\title{
The effects of GLP-1 analogues in obese, insulin-using type 2 diabetes in relation to eating behaviour
}

\author{
Stefanie Amarens de Boer ${ }^{1,2} \cdot$ Joop Daniel Lefrandt ${ }^{2} \cdot$ Japke Frida Petersen $^{1,2} \cdot$ \\ Hendrikus Hessel Boersma ${ }^{3}$ - Douwe Johannes Mulder ${ }^{2} \cdot$ Klaas Hoogenberg $^{1}$
}

Received: 10 June 2015/ Accepted: 7 November 2015/Published online: 23 November 2015

(C) The Author(s) 2015. This article is published with open access at Springerlink.com

\begin{abstract}
Background Glucagon-like peptide-1 receptor agonists (GLP-1 RA) added to insulin in type 2 diabetes patients have shown to lower body weight, improve glycaemic control and reduce total daily insulin dose in short term studies, although the individual response greatly varies. Objective To evaluate GLP-1 RA treatment on body weight, glycaemic control and total daily insulin dose in obese, insulin-using type 2 diabetes patients after 2 years follow-up in a real life setting and to explore a possible relation with eating behaviour. Setting The Martini Hospital and the University Medical Center in Groningen in the Netherlands. Methods Eligible patients were at least 18 years of age, were on insulin therapy and obese (BMI $>30 \mathrm{~kg} / \mathrm{m}^{2}$ ), started GLP-1 RA treatment. At baseline eating behaviour was classified according to the validated Dutch Eating Behaviour Questionnaire. A 2 years follow-up was performed. Main outcome measures Body weight, HbA1c and total daily insulin dose. Results 151 Patients started with exenatide or liraglutide. 120 patients
\end{abstract}

Electronic supplementary material The online version of this article (doi:10.1007/s11096-015-0219-8) contains supplementary material, which is available to authorized users.

Klaas Hoogenberg

HoogenK@mzh.nl

1 Department of Internal Medicine, Martini Hospital, Groningen, The Netherlands

2 Department of Vascular Medicine, University Medical Center Groningen, University of Groningen, Groningen, The Netherlands

3 Department of Clinical Pharmacy and Pharmacology, University Medical Center Groningen, University of Groningen, Groningen, The Netherlands completed the 2 years follow-up. From baseline to 2 years, body weight (mean $\pm \mathrm{SD}$ ) changed from $117.9 \pm 22.1$ to $107.9 \pm 22.9 \mathrm{~kg} \quad(P<0.0001)$, HbAlc (median, IQR) changed from $7.9(7.2-8.9)$ to $7.6(6.9-8.3) \%$ [63 (55-74) to $60(52-67) \mathrm{mmol} / \mathrm{mol}](P<0.0001)$, total daily insulin dose changed from $90(56-150)$ to $60(0-100)$ Units/day $(P<0.0001)$. Weight change differed between eating behaviour groups $(P<0.001)$ in which external eating behaviour $(n=17)$ resulted in the smallest decline $(-3.1 \%)$ and restrained $(n=41)$ in the greatest $(-10.3 \%)$ in comparison with emotional $(n=37$, $-8.5 \%)$ and indifferent $(n=25,-9.6 \%)$ eating behaviours. Conclusion Two year of GLP-1 RA treatment resulted in a sustained reduction of weight, $\mathrm{HbAlc}$ and total daily insulin dose in obese, insulin-using type 2 diabetes patients in a real life setting. Largest weight loss was achieved in patients with a predominant restraint eating pattern while a predominant external eating pattern resulted in the smallest weight reduction.

Keywords Diabetes mellitus - Eating behaviour - GLP-1 receptor agonist $\cdot$ Insulin therapy $\cdot$ Weight loss

\section{Impacts on practice}

- Addition of glucagon-like peptide-1 receptor agonists (GLP-1 RA) to obese, insulin-treated type 2 diabetes patients markedly reduces body weight, $\mathrm{HbA1c}$ and daily insulin doses or insulin discontinuation in a long term clinically based setting.

- Preexistent eating behavior modifies the amount of weight loss and assessment of eating behavior may help to identify those patients who will benefit most from GLP-1 RA. 


\section{Introduction}

Glucagon-like peptide-1 receptor agonists (GLP-1 RA) are used for weight loss and insulin dose reduction in obese insulin-using type 2 diabetic patients [1-7]. However, in daily practice the individual response of weight loss to GLP-1 RA varies greatly [8]. A recent Dutch cohort study [1] confirmed two earlier clinical based observational studies [2,3] of a more than expected weight reduction than generally reported in randomized controlled trials [47]. A high BMI and longer diabetes duration at start of treatment has been identified as predictors of greater weight loss [8] but other factors involved are unknown.

A plausible mechanism by which GLP-1 RA may induce weight loss is by suppressing appetite signalling in the brain and increasing satiety, leading to a reduced food intake $[9,10]$. GLP-1 receptors are present in the central nervous system suggesting direct actions of GLP-1 in the brain [11]. GLP-1 infusions can enhance satiety and reduce energy intake in type 2 diabetes patients [12]. Furthermore, GLP-1 RA attenuates binge eating in obese patients [13], suggesting a role of GLP-1 RA in certain eating types.

The psychology of eating distinguishes three main types of eating behaviours i.e. predominant external, emotional, and restrained eating patterns. External eaters are triggered in response to sensory stimuli irrespective of satiety [14]. Emotional eaters are driven by stress and emotions while the natural response would be to loose appetite [15]. Restrained eaters intentionally limit food intake to control weight. However, the self-imposed food restriction in restrained eaters is recognised by the body as true food shortage which goes into the starvation mode thus increasing hunger and lowering metabolic rate [16]. All these three eating behaviour patterns have been implicated in the risk of developing obesity [17]. A possible relation between eating patterns and response to GLP-1 RA has never been studied in patients with type 2 diabetes on insulin.

\section{Aim of the study}

To explore the role of eating behaviour on changes in weight, glycaemia and total daily insulin dose (TDD) in obese, insulin-using type 2 diabetes patients in clinical practise.

\section{Ethical approval}

The study was notified by the local ethics committee and was performed in accordance with the principles of the Declaration of Helsinki. All patients consented with the protocol including regular outpatient follow-up (FU) and the off-label use of GLP-1 RA with insulin.

\section{Methods}

\section{Study design and participants}

This prospective observational cohort study recruited patients at the Martini Hospital Groningen and the University Medical Center Groningen in the Netherlands. Eligible subjects were obese (BMI $>30 \mathrm{~kg} / \mathrm{m}^{2}$ ), insulinusing type 2 diabetes with a long-standing wish of weight reduction. They were $>18$ years of age, had a diabetes duration for $>1$ year and were receiving long-, intermediate or short-acting insulin (insulin glargine, insulin detemir, NPH insulin, regular insulin, insulin aspart, insulin lispro, or mixed insulin) with or without oral antidiabetic drugs (OAD). Patients were excluded if they had recurrent hypoglycaemia, a history of bariatric surgery, a history of pancreatitis, or had been treated with anti-obesity medication. The goals of GLP-1 RA treatment were weight loss and insulin dose reduction. The first patient started GLP-1 RA in January 2008 and the last patient started in January 2011. The study sample size was reflective of the number of eligible patients during this period.

\section{Study protocol}

GLP-1 RA treatment was initially given with exenatide, dose increased to $10 \mu \mathrm{g}$ twice daily, which was at the start of the study in January 2008 the only available GLP-1 RA in the Netherlands. In May 2009, liraglutide was the second GLP-1 RA marketed in the Netherlands and preferred by patients because of its once daily administration, dose increased to $1.8 \mathrm{mg}$ per day. At that time it was decided to study both GLP-1 RA with the intention to make a between GLP-1 RA drug comparison on outcomes.

No specific dietary restrictions were given for the study other than that all patients had received previous instructions and education on a healthy diet and lifestyle in accordance with the Dutch standards of care. FU was done at 6 weeks, 3, 6, 9, 12, 18 months, and after 2 years. At all study visits $\mathrm{HbAlc}(\%)$, body weight $(\mathrm{kg})$ and blood pressure were measured (mmHg), adherence to GLP-1 RA therapy, TDD (U/day), and OAD were documented. Adverse events were also noted including hypoglycaemic events. Hypoglycaemia was defined as major if the patient needed assistance for treatment of hypoglycaemia. At the start and after 2 years of FU serum creatinine (umol/l), triglycerides (mmol/l), HDL-, LDL-, and total cholesterol $(\mathrm{mmol} / \mathrm{l})$ were measured. 


\section{Dutch Eating Behaviour Questionnaire}

At baseline, eating behaviour was classified according to the validated Dutch Eating Behaviour Questionnaire (DEBQ) [18]. The DEBQ has a scale on restrained eating (e.g. "Do you try to eat less at mealtimes than you would like to eat?") and two separate scales on overeating tendency: emotional eating (e.g. "Do you have a desire to eat when you are irritated?") and external eating (e.g. "If food smells and looks good, do you eat more than usual?") [18]. Patients were classified according to their predominant eating pattern as defined by van Strien [18] as restrained, emotional, external or indifferent eaters.

\section{Outcome measures}

The primary outcome variable was change in weight after 2 years FU. Secondary outcome measures were change in HbA1c and TDD after 2 years FU. Safety parameters included: hypoglycaemia, nausea, vomiting, diarrhoea, and hospital admission.

\section{Statistical analysis}

Data are presented as numbers and percentage. Variables with a normal distribution are presented as mean \pm SD and otherwise as median and inter-quartile range (IQR). Statistical analyses were performed using the Statistical Package for Social Sciences version 20 (SPSS Inc., Chicago, Iln, USA). Variables at baseline and after 2 years of treatment were compared using the Students paired $t$ test and a Wilcoxon test were required. To compare groups, the Fisher exact or a one way ANOVA was used (or a Kurskall Wallis test if necessary), with logarithmic transformation for non-normally distributed variables and a post hoc Bonferroni for multiple comparisons if significant. To compare multivariate means between groups, a MANOVA was used. Backward multiple linear stepwise regression was used to identify variables independently $(P<0.100)$ associated with change in weight, HbA1c, and TDD. Logistic regression was used to identify variables independently associated with stopping insulin treatment. Since the duration of diabetes and BMI may be potential confounders, subgroup analyses were performed by dividing the study cohort into tertiles of these variables. A $P<0.05$ was considered statistically significant.

\section{Results}

One hundred and fifty-one obese, insulin-using type 2 diabetes patients were started on GLP-1 RA therapy. One hundred and twenty patients completed the 2 years of FU,
21 stopped $\leq 6$ months mainly due to side-effects (mostly gastrointestinal) and because they experienced no effects on weight and insulin dose. Nine patients were lost to FU, and one patient died due to myocardial infarction. The present analysis was done in those patients $(n=120)$ that completed 2 year FU after the initial prescription.

\section{Clinical characteristics}

Table 1 shows the clinical characteristics at baseline and after 2 years FU. Mean age was $58.4 \pm 8.1$ years, $52.5 \%$ were females, and the median diabetes duration was 10.0 (7.0-16.0) years. From baseline to 2 years, body weight $($ mean $\pm \mathrm{SD})$ changed from $117.9 \pm 22.1$ to $107.9 \pm$ $22.9 \mathrm{~kg}(P<0.0001), \mathrm{HbA} 1 \mathrm{c}$ (median, IQR) changed from $7.9(7.2-8.9)$ to $7.6(6.9-8.3) \mathrm{mmol} / \mathrm{l}[63(55-74)$ to 60 $(52-67) \mathrm{mmol} / \mathrm{mol}](P<0.0001)$, TDD changed from 90 $(56-150)$ to $60(0-100)$ Units/day $(P<0.0001)$ and $30 \%$ $(n=36)$ of the patients were able to stop insulin treatment. A total of 57 patients $(47.5 \%)$ experienced side effects, mostly gastrointestinal including nausea and vomiting $(n=45)$, diarrhoea $(n=3)$, constipation $(n=1)$. Two patients had to be hospitalized because of acute renal failure following severe dehydration due to a lack of intake. No severe hypoglycaemia occurred.

\section{Exenatide and liraglutide treatment}

Of the 120 patients completing the 2 years FU, 73 patients started with exenatide and 56 patients started with liraglutide. A total of 18 patients switched from exenatide to liraglutide when liraglutide became available and one patient switched from liraglutide to exenatide. Subgroup analysis revealed no differences in clinical characteristics of patients using exenatide or liraglutide, according to treatment at baseline and after 2 years FU (Supplemental table S1). Exenatide and liraglutide showed similar weight losses [exenatide (mean, SD) $-11.6 \pm 8.3 \mathrm{~kg}$ vs liraglutide $-8.8 \pm 7.5 \mathrm{~kg}, P=$ 0.058 ], similar reductions in $\mathrm{HbA1c}$ (exenatide $-0.54 \pm$ $1.4 \%$ vs liraglutide $-0.43 \pm 1.0 \%, P=0.641$ ), similar reductions in insulin dose [exenatide (median, IQR) -31 ( -60 to -7 ) U/day vs liraglutide -45 ( -63 to -15$) \mathrm{U} /$ day, $P=0.119$ ] all according to the treatment received at 2 years FU. Since weight loss, $\mathrm{HbA} 1 \mathrm{c}$ and insulin dose reduction were similar, the two GLP-1 treatments, exenatide and liraglutide, were combined in the further analysis.

\section{Change in weight}

The overall changes in weight according to different predefined groups (diabetes duration, BMI and eating behaviour) are given in Fig. 1a-c. There were no significant differences in weight changes among diabetes duration 
Table 1 Clinical characteristics at baseline and change after 2 years

\begin{tabular}{|c|c|c|c|}
\hline Characteristics & At baseline & After 2 years & $P$ value $^{\mathrm{a}}$ \\
\hline $\mathrm{N}$ & 120 & & \\
\hline Female (n) & $63(52.5 \%)$ & & \\
\hline Age (years) & $58.4(8.1)$ & & \\
\hline Diabetes duration (years) & $10(7.0-16.0)$ & & \\
\hline $\operatorname{BMI}\left(\mathrm{kg} / \mathrm{m}^{2}\right)$ & $39.5(6.5)$ & $36.1(6.2)$ & $<0.0001$ \\
\hline Weight (kg) & $117.9(22.1)$ & $107.9(21.9)$ & $<0.0001$ \\
\hline $\mathrm{HbA} 1 \mathrm{c}(\%)$ & $7.9(7.2-8.9)$ & $7.6(6.9-8.3)$ & $<0.0001$ \\
\hline $\mathrm{HbA1c}(\mathrm{mmol} / \mathrm{l})$ & $63(55-74)$ & $60(52-67)$ & \\
\hline Total cholesterol (mmol/l) & $4.0(3.6-4.6)$ & $3.8(3.4-4.6)$ & 0.011 \\
\hline HDL (mmol/l) & $1.1(0.9-1.3)$ & $1.1(0.9-1.3)$ & 0.834 \\
\hline LDL (mmol/l) & $2.1(1.6-2.5)$ & $2.1(1.6-2.5)$ & 0.601 \\
\hline Triglycerides (mmol/l) & $1.94(1.47-3.08)$ & $1.77(1.31-2.35)$ & $<0.0001$ \\
\hline Serum creatinine (umol/l) & $74.5(66.0-88.8)$ & $74.0(62.0-85.0)$ & 0.006 \\
\hline Systolic blood pressure $(\mathrm{mmHg})$ & $140.0(130-148)$ & $133.0(120-140)$ & $<0.0001$ \\
\hline Diastolic blood pressure $(\mathrm{mmHg})$ & $80.0(75.0-88.0)$ & $80.0(70.0-80.0)$ & $<0.0001$ \\
\hline \multicolumn{4}{|l|}{ Diabetes treatment } \\
\hline Insulin therapy (n) & $120(100 \%)$ & $84(69.4 \%)$ & $<0.0001$ \\
\hline Insulin dose (U/day) (all patients, $n=120$ ) & $90(56-150)$ & $60.0(0-100)$ & $<0.0001$ \\
\hline Insulin dose (U/kg/day) (all patients, $\mathrm{n}=120)$ & $0.75(0.47-1.33)$ & $0.54(0.00-0.87)$ & $<0.0001$ \\
\hline Insulin dose (U/day) (patients using insulin after 2 years, $n=84$ ) & $119(73-182)$ & $80(55-124)$ & $<0.0001$ \\
\hline Insulin dose (U/kg/day) (patients using insulin after 2 years, $n=84$ ) & $1.01(0.65-1.47)$ & $0.71(0.53-1.11)$ & $<0.0001$ \\
\hline \multicolumn{4}{|l|}{ Oral therapy } \\
\hline No oral therapy (n) & $13(10.8 \%)$ & $16(13.3 \%)$ & 0.453 \\
\hline 1 oral therapy $(\mathrm{n})$ & $80(66.7 \%)$ & $86(71.7 \%)$ & 0.307 \\
\hline 2 oral therapy $(n)$ & $27(22.5 \%)$ & $18(15 \%)$ & 0.049 \\
\hline \multicolumn{4}{|l|}{ GLP-1 treatment } \\
\hline Exenatide (n) & $73(60.8 \%)$ & $56(46.7 \%)$ & \\
\hline Liraglutide (n) & $47(39.2 \%)$ & $64(53.3 \%)$ & \\
\hline
\end{tabular}

Data are expressed as number (\%) or means (SD) or if not normal distributed as median IQR

${ }^{a} P<0.05$ indicates statistical significance

tertiles. Across baseline BMI tertiles, a greater weight loss was observed in patients with a higher baseline BMI $\left(36-40\right.$ and $>40 \mathrm{~kg} / \mathrm{m}^{2}$ ) which was significant at 9 months $(P=0.03), 12$ months $(P=0.025), 18$ months $(P=$ $0.010)$ and 2 years $\mathrm{FU}(P=0.021)$. According to eating behaviour, the smallest decline was observed in external eaters which was significant at 9 months $(P=0.032)$, 12 months $(P=0.010), 18$ months $\quad(P=0.001)$, and 2 years FU $(P=0.001)$.

Multiple linear stepwise regression analysis was used to identify the predictors of the change in weight at 2 years FU. Only baseline BMI, beta (95 \% CI) $-0.230(-0.399$ to $-0.062, P=0.008)$ was significantly associated with weight loss. Sex, age, baseline HbA1c, baseline TDD, and diabetes duration were not significant and were excluded from the model.

\section{Glycaemic control}

The overall change in HbA1c (geometric mean and $95 \%$ $\mathrm{CI}$ ) according to the predefined subgroups are given in Fig. 1d-f. Across diabetes duration tertiles, a lower HbA1c was observed in patients with a shorter diabetes duration ( $<8$ years) which was significant at 9 months $(P=0.001)$, 12 months $(P=0.003), 18$ months $(P=0.001)$, and 2 years $\mathrm{FU}(P=0.002)$. There were no significant differences in HbA1c among baseline BMI tertiles and eating behaviour groups.

Multiple linear stepwise regression analysis was used to identify the factors (eating behaviour excluded) associated with change in $\mathrm{HbA} 1 \mathrm{c}$ at 2 years FU. Only baseline HbA1c beta $(95 \% \mathrm{CI})-0.587(-0.733$ to $-0.441, P<0.0001)$, and diabetes duration beta $0.191(0.043-0.338, P=0.011)$ 
A Change in weight by duration of diabetes

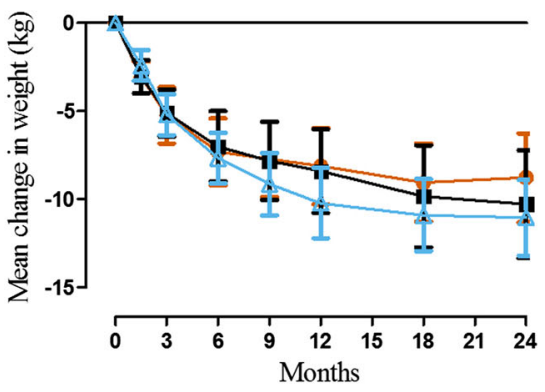

B Change in weight by baseline BMI

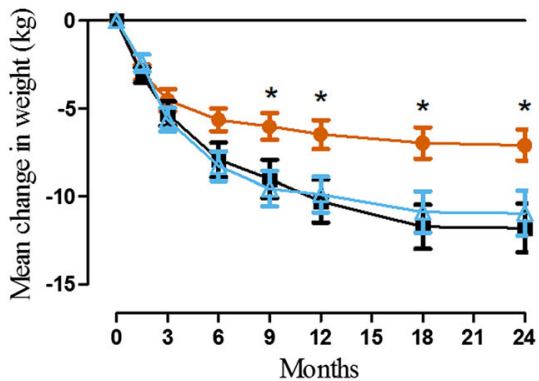

C Change in weight by eating behavior

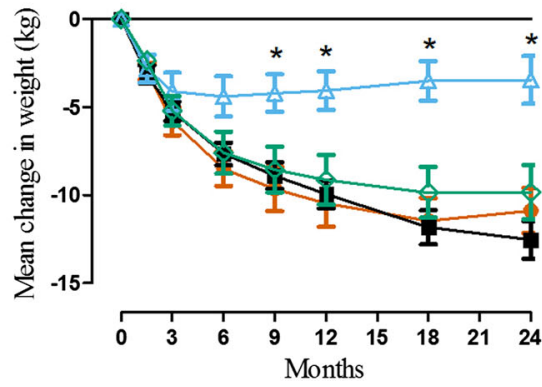

Fig. 1 Change in weight $(\mathrm{kg})$ according to duration of diabetes (a), baseline BMI (b) and eating behaviour group (c); HbA1c (\%) according to duration of diabetes (d), baseline BMI (e) and eating behaviour group (f) in the patients $(\mathrm{n}=120)$ who completed the

were independently associated with changes of $\mathrm{HbA1c}$. Sex, age, baseline BMI, and baseline TDD were not significant and were excluded from the model. Moreover, there was no relation between change in weight $(\mathrm{kg})$ and change in $\mathrm{HbA} 1 \mathrm{c}(\%)$ at 2 years FU, Pearson correlation $0.013, P=0.887$.

\section{Insulin dose}

The decrease in TDD throughout the 2 years of FU (Table 1) across the diabetes duration tertiles, was lower in patients with a shorter diabetes duration $(<8$ years $)$ $(P<0.0001$ at every point of FU). Across baseline BMI tertiles, patients with a lower BMI $\left(36<\mathrm{kg} / \mathrm{m}^{2}\right)$ used significantly $(P<0.05)$ less Units/day at every point of
AlC by duration of diabetes


E A1C by baseline BMI
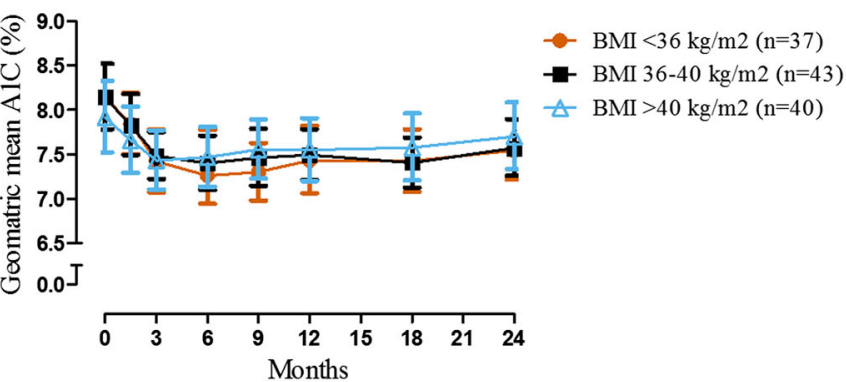

F AlC by eating behavior
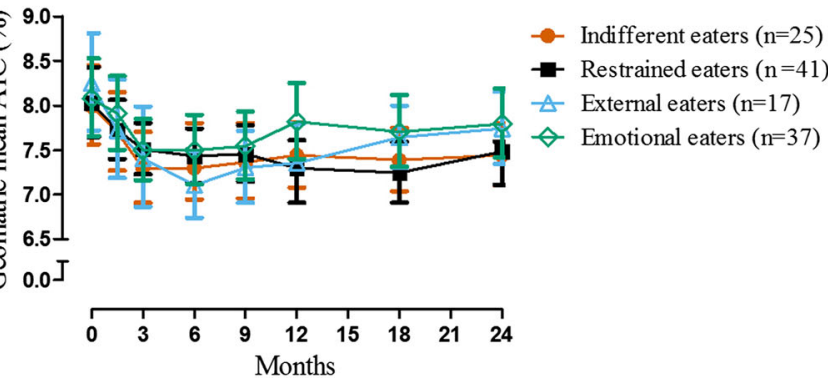

2 years follow-up. Data are presented as mean change in weight (SEM) and geometric mean $(95 \% \mathrm{CI})$. Asterisk denotes $P<0.05$ to $P<0.01$ from other groups

FU. The change in TDD did not differ between baseline BMI tertiles and eating behaviour groups.

Multiple linear stepwise regression analysis was used to identify predictors (except for eating behaviour) of the change in TDD at 2 years FU. Only baseline TDD, beta $(95 \% \mathrm{CI})-0.450(-0.279$ to $-0.622, P<0.0001)$ and diabetes duration, beta $0.176(0.005-0.347, P=0.044)$ were significant associated with changes of TDD, if tested stepwise. Sex, age, baseline HbA1c, and baseline BMI were not significant and were excluded from the model. In the logistic regression analysis, only diabetes duration (years) with an odds-ratio $(95 \% \quad \mathrm{CI})$ of 0.893 $(0.814-0.979, P=0.016)$ and TDD (Units) with an oddsratio of $0.976(0.964-0.988, P<0.0001)$ were associated with stopping insulin treatment, whereas sex, age, baseline 
HbAlc, and baseline BMI were not independently associated.

\section{Eating behaviour}

In each group of eating behaviour, body weight was significantly reduced after 2 years FU: external $(n=17)$ $117.8 \pm 18.7 \mathrm{~kg}$ to $114.4 \pm 20.4 \mathrm{~kg}(-3.1 \%, P<0.022)$, emotional $(n=37) 113.8 \pm 19.7 \mathrm{~kg}$ to $103.9 \pm 19.0 \mathrm{~kg}$ $(-8.5 \%, P<0.001)$, restrained $(n=41) 123.8 \pm 22.7 \mathrm{~kg}$ to $111.3 \pm 22.5 \mathrm{~kg} \quad(-10.3 \%, P<0.001)$, indifferent $(n=25) 114.6 \pm 25.4 \mathrm{~kg}$ to $103.8 \pm 24.8 \mathrm{~kg}(-9.6 \%$, $P<0.001)$. Weight change at 2 years FU differed significantly between types of eating behaviour (Fig. 2, $P<0.001)$. After correction for differences between different types of eating behaviour (Supplemental table S1) in baseline BMI, diabetes duration, baseline HbA1c, and baseline TDD, the differences in change in weight at 2 years FU remained significant (MANOVA, $P<0.001$ ). Changes in HbA1c and TDD at 2 years FU did not differ significantly between types of eating behaviour. If tested with a one way ANOVA and post hoc Bonferroni predominant external eaters showed less change in weight which was significant at 9 months $(P=0.032), 12$ months $(P=0.010), 18$ months $(P=0.010)$ and 2 years $\mathrm{FU}$ $(P=0.010)$ compared to emotional, restrained and indifferent predominant eaters.

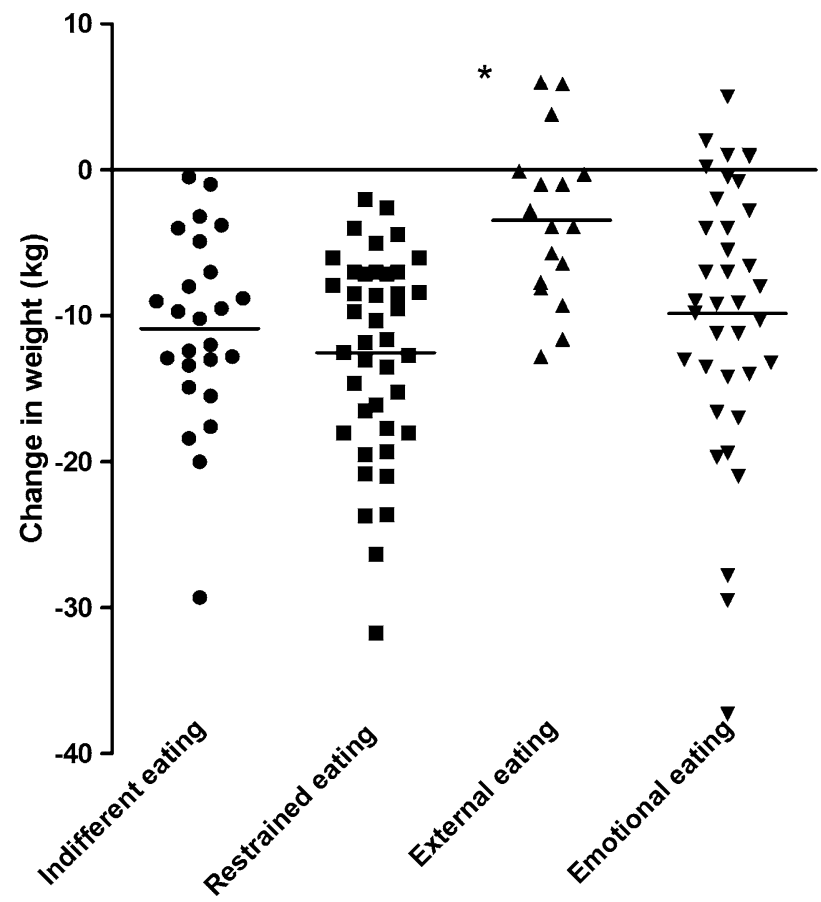

Fig. 2 Individual changes in weight at 2 years of follow-up according to eating behaviour category. Asterisk denotes $P<0.05$ from other groups

\section{Discussion}

The current study demonstrated that GLP-1 RA given to obese, insulin-using type 2 diabetes patients resulted in a marked weight loss, improved glycaemic control and considerably reduced daily insulin doses. These changes were most prominent during the first 3 months of GLP-1 RA treatment, then gradually tapered and sustained during 2 years of follow-up. Thereby these findings confirm and expand other clinical based cohort studies [1-3] that showed a more than average weight loss on GLP-1 RA than generally reported [4-7]. In addition, we found that pre-existing eating behaviour influenced GLP1 RA induced weight loss, which was obvious in restrained and indifferent eaters and mitigated in patients with an external eating trait.

GLP-1 RA have consistently shown to reduce weight and improve glycaemic control in insulin-using type 2 diabetes patients, however variable responses have been reported [4-7]. Two systematic reviews mentioned a -0.9 to $-5.6 \mathrm{~kg}$ and $\mathrm{a}-1.5$ to $-4.9 \mathrm{~kg}(-3.2 \mathrm{~kg}$ on average) weight loss when GLP-1 RA was added to insulin in type 2 diabetes patients [6,7]. Our study demonstrated a much larger weight loss that corresponded with the amount of weight reduction of $-6.5( \pm 0.8) \mathrm{kg}$ at 26 weeks [2], -12.8 $( \pm 7.5) \mathrm{kg}[3]$ and $-14.3( \pm 9.5) \mathrm{kg}$ [1] at 52 weeks at the consecutive time points in our study.

The GLP-1 RA therapy was given either with exenatide, an exendin-based GLP-1 receptor agonist, or with liraglutide, a human GLP-1 analogue, for reasons connected to the clinical based observational nature of our study (see "Methods" section). A comparison of the efficacy of both treatment options disclosed no meaningful differences in outcome of our study. This is in conjunction with the RCT that head-to-head compared exenatide and liraglutide (LEAD-6 trial) [19] showing similar weight loss of both drugs. The LEAD-6 trial found larger HbA1c reductions with liraglutide than with exenatide but in a quite different type 2 diabetic patient population only using OAD treatment and no insulin.

Little is known about the factors that determine the amount of weight loss by GLP-1 RA treatment. In a post hoc analysis on a previous RCT, the greatest reductions in weight and also $\mathrm{HbA1c}$ were seen in the more obese patients $\left(\mathrm{BMI}>30 \mathrm{~kg} / \mathrm{m}^{2}\right.$ ) and the longest diabetes duration $(>13$ years) [8]. In conjunction, we found the greatest weight reductions in the most obese patients $\left(\mathrm{BMI}>36 \mathrm{~kg} / \mathrm{m}^{2}\right)$ and a non-significant tendency for a longer diabetes duration ( $>13$ years). Thus the substantial weight loss that we and the few other studies observed, may have resulted from higher baseline BMI that amply exceeded $35 \mathrm{~kg} / \mathrm{m}^{2}$ in all [1-3]. A confounding factor may have been the selection of patients as we included those in 
whom overweight complicated their diabetes with weight reduction as the primary treatment goal. Therefore a selection bias may have occurred as some patients who did not experience an early clinical benefit from GLP-1 RA discontinued the study. Since our study was observational in nature with no control group, it is difficult to determine the exact weight loss attributable to the GLP-1 RA treatment. Notwithstanding that the currently observed weight loss was that what genuinely can be achieved in outpatient clinical setting, as recently reported [1].

To our knowledge, this is one of the first studies that investigated eating behaviour in relation to efficacy of GLP$1 \mathrm{RA}$ administration showing that restrained and indifferent eaters obviously lost weight and external eaters had the smallest reductions in weight loss. There is only one small Japanese study in obese type 2 diabetes patients $(\mathrm{n}=16)$ that assessed eating scores and traits in relation to the GLP-1 RA, showing that amount of weight loss correlated with a promptly reduced score for the sense of hunger that showed an interaction with external eating behaviour [20].

There are a few studies that might provide a pathophysiological basis by which external eating mitigated weight reduction. First, in an functional magnetic resonance imaging (fMRI) study healthy obese men were treated with exenatide or saline infusion in a blinded fashion and shown food pictures (external eating stimulus). Only in those subjects who showed $\geq 10 \%$ reduction in caloric intake (responders), effects of exenatide were observed on the fMRI in the hypothalamus [21]. Second, in another fMRI study the brain responses to food pictures were higher in obese non-diabetic and type 2 diabetes than lean subjects [10]. GLP-1 RA administration decreased the brain responses to food pictures and food intake in the obese non-diabetic and type 2 diabetes subjects and a lower reduction in food picture-induced brain activation (external stimulus) led to a smaller decrease in food intake [10]. In terms of external eating behaviour this means that persistent brain activation was marginally diminished by GLP-1 RA and did not change food intake. Hence from these experimental studies, we suppose that a higher proportion of external eaters could have diminished or lacking effects of GLP1-RA on the brain.

The DEBQ is a widely used questionnaire that was developed to measure the three eating behaviour styles which are generally accepted as the psychological basis of overeating. It has been shown that patients with obesity, anorexia nervosa, and healthy controls all have a different eating behaviour as measured by the DEBQ [22]. Interestingly, reduced GLP-1 levels in bulimia nervosa have been associated with binge-eating episodes [23]. As bingeeating is common in restrained and in emotional eaters, this may give an indication why these eating behaviour patterns in our study benefited from GLP-1 RA treatment.
The diabetes treatment was individually tailored by the treating physicians (JDL, KH, KMT, SHJD). Despite the limitation that no strict treatment protocol for glycaemic control was used, net glycaemia improved, TDD decreased with an average of $30 \mathrm{U} /$ day with $30 \%$ of patients stopping insulin and no severe episodes of hypoglycaemia. Thus this clinical based study provided an impression of the expected benefit on $\mathrm{HbAlc}$ and insulin dose reduction that can be achieved by GLP1 RA treatment in daily practise.

\section{Conclusion}

This observational cohort study showed that GLP-1 RA treatment resulted in a sustained reduction of weight, HbA1c and TDD in obese insulin-using type 2 diabetes patients in a real life setting. Largest weight loss was achieved in patients with a predominant restraint eating pattern while a predominant external eating pattern resulted in the smallest weight reduction. The finding that pre-existing eating behaviour modulated the effects of GLP1-RA on weight loss deserves further study.

Acknowledgments We thank the treating physicians K.M. van Tol and S.H.J. Donders, Martini Hospital, the Netherlands, that apart from the authors JDL and KH kindly helped with performing the study during their daily clinical practise. We thank E. Vredeveld for her support with the collection of data.

Funding There was no funding for this study.

Conflicts of interest $\mathrm{KH}$ received educational grants and lecture fees from Novo Nordisk and Lilly. No other potential conflicts of interest relevant to this article were reported.

Open Access This article is distributed under the terms of the Creative Commons Attribution 4.0 International License (http://crea tivecommons.org/licenses/by/4.0/), which permits unrestricted use, distribution, and reproduction in any medium, provided you give appropriate credit to the original author(s) and the source, provide a link to the Creative Commons license, and indicate if changes were made.

\section{References}

1. Van Velsen EFS, Lamers J, Blok V, Van Leendert RJM, KiewietKemper RM. A prospective study of concomitant GLP-1 analogue and insulin use in type 2 diabetes in clinical practice. Neth $\mathrm{J}$ Med. 2014;72:523-7.

2. Viswanathan P, Chaudhuri A, Bhatia R, Al-Atrash F, Mohanty P, Dandona P. Exenatide therapy in obese patients with type 2 diabetes mellitus treated with insulin. Endocr Pract. 2007;13: 444-50.

3. Nayak UA, Govindan J, Baskar V, Kalupahana D, Singh BM. Exenatide therapy in insulin-treated type 2 diabetes and obesity. QJM. 2010;103:687-94. 
4. Vilsboll T, Christensen M, Junker AE, Knop FK, Gluud LL. Effects of glucagon-like peptide-1 receptor agonists on weight loss: systematic review and meta-analyses of randomised controlled trials. BMJ. 2012;344:d7771.

5. Drucker DJ, Nauck MA. The incretin system: glucagon-like peptide-1 receptor agonists and dipeptidyl peptidase-4 inhibitors in type 2 diabetes. Lancet. 2006;368:1696-705.

6. Goldenberg R. Insulin plus incretin agent combination therapy in type 2 diabetes: a systematic review. Curr Med Res Opin. 2014;30:431-45.

7. Eng C, Kramer CK, Zinman B, Retnakaran R. Glucagon-like peptide-1 receptor agonist and basal insulin combination treatment for the management of type 2 diabetes: a systematic review and meta-analysis. Lancet. 2014;384:2228-34.

8. Rosenstock J, Shenouda SK, Bergenstal RM, Buse JB, Glass LC, Heilmann CR, et al. Baseline factors associated with glycemic control and weight loss when exenatide twice daily is added to optimized insulin glargine in patients with type 2 diabetes. Diabetes Care. 2012;35:955-8.

9. Zander M, Madsbad S, Madsen JL, Holst JJ. Effect of 6-week course of glucagon-like peptide 1 on glycaemic control, insulin sensitivity, and beta-cell function in type 2 diabetes: a parallelgroup study. Lancet. 2002;359:824-30.

10. Van Bloemendaal L, IJzerman RG, Ten Kulve JS, Barkhof F, Konrad RJ, Drent ML, Veltman DJ, Diamant M. GLP-1 receptor activation modulates appetite- and reward-related brain areas in humans. Diabetes. 2014;63:4186-96.

11. Yamamoto H, Lee CE, Marcus JN, Williams TD, Overton JM, Lopez ME, et al. Glucagon-like peptide-1 receptor stimulation increases blood pressure and heart rate and activates autonomic regulatory neurons. J Clin Invest. 2002;110:43-52.

12. Toft-Nielsen MB, Madsbad S, Holst JJ. Continuous subcutaneous infusion of glucagon-like peptide 1 lowers plasma glucose and reduces appetite in type 2 diabetic patients. Diabetes Care. 1999;22:1137-43.

13. Robert SA, Ghani RA, Zainuddin S, Shah SA, Mohamud WNW, Kamaruddin NA. The influence of a GLP-1 analogue, liraglutide on binge eating behavior among obese healthy participants. In: Data presented at: Annual Scientific Meeting of the Australian and New Zealand Obesity Society (ANZOS), Melbourne, Australia; Oct 17-19 2013.

14. Herman CP, Polivy J. External cues in the control of food intake in humans: the sensory-normative distinction. Physiol Behav. 2008;94:722-8.

15. Van Strien T, van der Zwaluw CS, Engels RC. Emotional eating in adolescents: a gene (SLC6A4/5-HTT) —depressive feelings interaction analysis. J Psychiatr Res. 2010;44:1035-42.

16. Herman CP, Polivy J. Anxiety, restraint, and eating behavior. J Abnorm Psychol. 1975;84:66-72.

17. Chaput JP, Leblanc C, Perusse L, Despres JP, Bouchard C, Tremblay A. Risk factors for adult overweight and obesity in the Quebec Family Study: Have we been barking up the wrong tree? Obesity (Silver Spring). 2009;17:1964-70.

18. Van Strien T, Frijters J, Bergers G, Defares P. Nederlandse Vragenlijst voor eetgedrag (NVE). Handleiding [Dutch Eating Behavior Questionnaire Manual]. Lisse; 1986.

19. Buse JB, Rosenstock J, Sesti G, Schmidt WE, Montanya E, Brett $\mathrm{JH}$, et al. Liraglutide once a day versus exenatide twice a day for type 2 diabetes: a 26-week randomised, parallel-group, multinational, open-label trial (LEAD-6). Lancet. 2009;374:39-47.

20. Fujishima Y, Maeda N, Inoue K, Kashine S, Nishizawa H, Hirata A, et al. Efficacy of liraglutide, a glucagon-like peptide-1 (GLP1) analogue, on body weight, eating behavior, and glycemic control, in Japanese obese type 2 diabetes. Cardiovasc Diabetol. 2012;11:107.

21. Schlogl H, Kabisch S, Horstmann A, Lohmann G, Muller K, Lepsien $\mathbf{J}$, et al. Exenatide-induced reduction in energy intake is associated with increase in hypothalamic connectivity. Diabetes Care. 2013;36:1933-40.

22. Banos RM, Cebolla A, Moragrega I, Van Strien T, FernandezAranda F, Aguera Z, et al. Relationship between eating styles and temperament in an Anorexia Nervosa, Healthy Control, and Morbid Obesity female sample. Appetite. 2014;76:76-83.

23. Dossat AM, Bodell LP, Williams DL, Eckel LA, Keel PK. Preliminary examination of glucagon-like peptide-1 levels in women with purging disorder and bulimia nervosa. Int $\mathrm{J}$ Eat Disord. 2015;48:199-205. 